\title{
Solid dispersions: a technology for improving bioavailability
}

\begin{abstract}
An unending challenge in pharmaceutical industry is related to poor solubility of maximum drugs. To overcome this problem various technologies have been developed but none appears to be a promising one. Solid dispersion is a solubilization technology emphasizing basically on drug-polymer two component systems in which drug dispersion and its stabilization is the key for formulation development. Therefore this technology has been realized as extremely useful tool in improving the dissolution properties of poorly water-soluble drugs and in recent years, a great deal of knowledge has been accumulated about solid dispersion, but their commercial application is limited. This review summarizes our current understanding of various methods used for the preparation of solid dispersions emphasizing the elementary aspects of this significant technology.
\end{abstract}

Keywords: solid dispersions, poorly soluble drug, solid solution, amorphous state, bioavailability
Volume 8 Issue 4 - 2019

\author{
Kiran Singh Sharma, Jagannath Sahoo, Seema \\ Agrawal, Asha Kumari
}

KIET Group of Institutions, KIET School of Pharmacy, India

Correspondence: Kiran Singh Sharma, Assistant Professor, KIET Group of Institutions, KIET School of Pharmacy, 13 km stone, Ghaziabad-meerut road, Ghaziabad 201206 (UP), India, Email kiran.sharma@kiet.edu

Received: January 30, 2019 | Published: July 02, 2019

\section{Introduction}

Modern drug discovery techniques, with advances in combinatorial chemistry and high throughput screening, continue to fill drug development pipelines with a high number of poorly soluble New Chemical Entities (NCEs). It is estimated that over the years about $40 \%-70 \%$ of NCEs are poorly water soluble and large number of scientists are engage in invention of NCEs and the success rate is poor. A drug with poor aqueous solubility will typically exhibit dissolution rate limited absorption, and a drug with poor membrane permeability will typically exhibit permeation rate limited absorption. Hence, two areas of pharmaceutical research that focus on improving the oral bioavailability of active agents include: enhancing solubility and dissolution rate of poorly water soluble drug and enhancing permeability of poorly permeable drugs. ${ }^{1}$

The main possibilities for improving dissolution is to increase the surface area available for dissolution by decreasing the particle size of the solid compound and/or by optimizing the wetting characteristics of the compound surface, to decrease the boundary layer thickness, to ensure sink conditions for dissolution and, last but definitely not least, to improve the apparent solubility of the drug under physiologically relevant conditions. Of these possibilities, changes in the hydrodynamics are difficult to invoke in vivo and the maintenance of sink conditions will depend on how permeable the gastrointestinal mucosa is to the compound as well as on the composition and volume of the lumenal fluids. Although some research effort has been directed towards permeability enhancement using appropriate excipient, results to date have not been particularly encouraging. ${ }^{2}$

Administration of the drug in the fed state may be an option to improve the dissolution rate and also to increase the time available for dissolution; the likely magnitude of the food effect can be forecasted from dissolution tests in biorelevant media.

In the Biopharmaceutical Classification System (BCS) drugs with low aqueous solubility and high membrane permeability are categorized as Class II drugs. Therefore, solid dispersion technologies are particularly promising for improving the oral absorption and bioavailability of BCS Class II drugs. ${ }^{3}$ The basic principle involved in enhancing the poor solubility of drug with solid dispersion includes complete removal of drug crystalline structure and its molecular dispersion in a hydrophilic polymeric carrier.

When the solid dispersion is exposed to aqueous media, the carrier dissolves and the drug releases as fine colloidal particles. This increases surface area of dissolution rate and hence bioavailability of poorly water soluble drugs. Drug in soluble hydrophilic carrier improves the dissolution rate by reducing particle size and increasing the particle porosity. Therefore by improving the drug release profile of these drugs, it is possible to enhance their bioavailability and reduce side effects. ${ }^{4}$

\section{Advantages of solid dispersion}

Improving drug bioavailability by changing their water solubility has been possible by chemical or formulation approaches.Chemical approaches to improving bioavailability without changing the active target can be achieved by salt formation or by incorporating polar or ionizable groups in the main drug structure, resulting in the formation of a pro-drug. Solid dispersions appear to be a better approach to improve drug solubility than these techniques, because they are easier to produce and more applicable. ${ }^{5}$

In molecular dispersions, solid dispersions represent the last state on particle size reduction, and after carrier dissolution the drug is molecularly dispersed in the dissolution medium. Solid dispersions apply this principle to drug release by creating a mixture of a poorly water soluble drug and highly soluble carriers. A high surface area is formed, resulting in an increased dissolution rate and, consequently, improved bioavailability. ${ }^{6}$ Solid dispersions also provides particles with improved wettability as it was observed that even carriers without any surface activity, such as urea improved drug wettability. Carriers with surface activity, such as cholic acid and bile salts, when used, can significantly increase the wettability property of drug.

Particles in solid dispersions have been found to have a higher degree of porosity. The increase in porosity also depends on the carrier properties; for instance, solid dispersions containing linear polymers 
produce larger and more porous particles than those containing reticular polymers and, therefore, result in a higher dissolution rate. The increased porosity of solid dispersion particles also hastens the drug release profile. ${ }^{7}$

Poorly water soluble crystalline drugs, when in the amorphous state tend to have higher solubility. The enhancement of drug release can usually be achieved using the drug in its amorphous state, because no energy is required to break up the crystal lattice during the dissolution process. In solid dispersions, drugs are presented as supersaturated solutions after system dissolution, and it is speculated that, if drugs precipitate, it is as a metastable polymorphic form with higher solubility than the most stable crystal form. For drugs with low crystal energy (low melting temperature or heat of fusion), the amorphous composition is primarily dictated by the difference in melting temperature between drug and carrier. ${ }^{8}$ For drugs with high crystal energy, higher amorphous compositions can be obtained by choosing carriers, which exhibit specific interactions with them. Drug is formulated with hydrophilic carrier as a solid dispersion to increase its aqueous solubility and dissolution. Then superdisintegrant (e.g. croscarmellose sodium) is used in tablet formulation to achieve rapid disintegration of tablets prepared by wet granulation method. These rapidly disintegrating tablets can be used as an alternative to parenteral therapy enabling patient for self-medication even without the aid of water. 9

\section{Disadvantages of solid dispersions}

Despite extensive expertise with solid dispersions, they are not broadly used in commercial products, mainly because of the possibility of the change of amorphous state to crystalline state during processing (mechanical stress) or storage (temperature and humidity stress).

The effect of moisture on the storage stability of amorphous pharmaceuticals is also a significant concern, because it may increase drug mobility and promote drug crystallization. Moreover, most of the polymers used in solid dispersions can absorb moisture, which may result in phase separation, crystal growth or conversion from the amorphous to the crystalline state or from a metastable crystalline form to a more stable structure during storage. This may result in decreased solubility and dissolution rate. Therefore, exploitation of the full potential of amorphous solids requires their stabilization in solid state, as well as during in vivo performance.

Another drawback of solid dispersions is their poor scale-up for the purposes of manufacturing. Strategies to overcome the manufacturing process drawbacks will be discussed later. ${ }^{10}$

\section{Classification of solid dispersions}

Depending on the molecular arrangement, solid dispersions can be of the following types:

Eutectic mixtures: solid eutectic mixtures are usually prepared by rapidly cooling the co-melt of the two components in order to obtain a physical mixture of very fine crystals of the two components. Solid solutions: Depending on the miscibility, the two types of solid solutions are:

Continuous solid solutions: In continuous solid solutions, the components are miscible in all proportions i.e. the bonding strength between the components is stronger than the bonding between the individual component.
Discontinuous solid solutions: In discontinuous solid solutions, the solubility of each of the component in the other component is limited in nature. ${ }^{11}$

\section{Solid solutions}

Depending on the distribution of the solvates in the solvent, solid solutions can be of two types:

Substitution crystalline solution: These are those solid solutions which have a crystalline structure, the solute molecules substitute for the solvent molecules in the crystal lattice.

Interstitial crystalline solid solution: These are those solid solutions in which the dissolved molecules occupy the interstitial spaces between the solvent molecules in the crystal lattice.

Amorphous solid solutions: In amorphous solid solutions, the solute molecules are dispersed molecularly but irregularly within the amorphous solvent.

Glass solutions and glass suspension: A glass solution is a homogenous system in which the solute dissolves in the glassy solvent. The glassy state is characterized by transparency and brittleness below the glass transition temperature. The term glass refers to a pure chemical or a mixture of pure chemicals in the glassy state. ${ }^{12}$

\section{Classification of solid dispersion on the basis of recent advancement}

First generation solid dispersion: These solid dispersions are prepared by using crystalline carriers. Urea and sugars were the first crystalline carriers that were used in the preparation of solid dispersions. These have a disadvantage of being thermodynamically unstable and they do not release drug at a faster rate.

Second generation solid dispersion: These solid dispersions are prepared using amorphous carriers instead of crystalline carriers. The drug is molecularly dispersed in the polymeric carrier. The polymeric carriers are divided into two groups:

a. Synthetic polymer-povidone, polyethylene glycols and polymethacrylates.

b. Natural polymers - hydroxypropylmethylcellulose, ethyl cellulose, starch derivatives like cyclodextrin.

Third generation solid dispersion: These solid dispersions contain a surfactant carrier, or a mixture of amorphous polymers and surfactants as carriers. These achieve the highest degree of bioavailability for the drugs that are having poor solubility. The surfactants being used in the third generation solid dispersion are such as inulin, poloxamer 407 etc. ${ }^{13}$

\section{Polymers}

A wide variety of polymers are available which have tremendous potential in the area of solid dispersions.

\section{Polyethylene glycol (PEG)}

These are compounds are obtained from a reaction of ethylene glycol with ethylene oxide. PEGs whose molecular weight is above 300000 are commonly termed as polyethylene oxides. For the manufacture of solid dispersions and solutions, PEGs with molecular weights of 1500-20,000 are usually employed. As the MW rises, so 
does the viscosity of the PEG. At MW of up to 600, PEGs are fluid, in the range $800-1500$ they have a consistency that is best described as vaseline-like, from 2000 to 6000 they are waxy and those with MW of 20,000 and above form hard, brittle crystals at room temperature. Their solubility in water is generally good, but reduces with MW. A meticulous advantage of PEGs for the solid dispersions is that they have good solubility in numerous organic solvents. The melting point of the PEGs of interest with an average molecular weight of 4600 (range $4400-4800$ ) is $57-61^{\circ} \mathrm{C}$ and with an average molecular weight of 6000 (range $5000-7000$ ) $60-63^{\circ} \mathrm{C}$

\section{Polyvinylpyrrolidone (PVP)}

PVP molecular weight ranges from 2500 to 3000000 . It is having solubility in solvents like water, ethanol, chloroform and isopropyl alcohol. PVP gets decomposed at high temperature therefore it is not suitable for preparation of solid dispersions prepared by melt method because melting takes place at a very high temperature. PVP can be classified according to the $\mathrm{K}$ value, which is calculated using Fikentscher's equation. The temperature of a given PVP is dependent not only on its MW but also on the moisture content. In general, the glass transition temperature (Tg) is high; for example, PVP K25 has a $\mathrm{Tg}$ of $1558^{\circ} \mathrm{C}$. For this reason PVPs have only restricted application for the preparation of solid dispersions by the hot melt method. Due to their excellent solubility in an ample variety of organic solvents, they are mostly suitable for the preparation of solid dispersions by the solvent method. ${ }^{14}$

\section{Cellulose derivatives}

Hydroxypropylmethylcellulose (HMPC): HPMCs are mixed ethers of cellulose, in which $16.5-30 \%$ of the hydroxyl groups are methylated and $4-32 \%$ is derivatized with hydroxypropyl groups. The molecular weight of the HPMCs ranges from about 10000 to 1500000 and they are soluble in water and mixtures of ethanol with dichloromethane and methanol with dichloromethane.

Hydroxypropylcellulose (HPC): Hydroxypropylcellulose (HPC) exhibits good solubility in a range of solvents, including water (up till 400C), ethanol, methanol and chloroform. The average MW of the HPCs ranges from 37000 (Type SSL) to 1150000 (Type H). ${ }^{4}$

Carboxymethylethylcellulose (CMEC): CMEC also belongs to the cellulose ethers, but unlike many of the others it is resistant to dissolution under gastric (acidic) conditions. It dissolves readily at $\mathrm{pH}$ values above 5-6, with lowest dissolution $\mathrm{pH}$ being dependent on the grade of the CMEC. CMECs also dissolve readily in acetone, isopropanol $70 \%$, ethanol $60 \%$ and $1: 1$ mixtures of dichloromethane and ethanol. ${ }^{15}$

Hydroxypropylmethylcellulose phthalate (HPMCP): HPMCPs are cellulose esters which are often used as enteric coatings. Depending on the grade, they dissolve first at pH 5 (HP 50) or $\mathrm{pH} 5.5$ (HP 55). They are having a type-dependent solubility in organic solvents. The dissolution rate of griseofulvin at $\mathrm{pH} 6.8$ could be greatly enhanced by incorporating it in a coevaporate of HPMCP. ${ }^{16}$

Polyacrylates and polymethacrylates: Polyacrylates and polymethacrylates are glassy substances that are produced by the polymerization of acrylic and methacrylic acid, and derivatives of these polymers such as esters amides and nitriles. In pharmaceuticals they are mainly used as coatings to change the release of the drug from the dosage form.

Phospholipids: The complexity of glycerides advances by modification of the terminal hydroxyl with phosphate linked head groups to form phospholipids, common phospholipid head groups include choline, ethanolamine, serine, inositol and inositol phosphate, and glycerol esters. As with the triglycerides, numerous species are possible by various combinations of different head groups and fatty acyl substitution at the first and second positions of the glycerol backbone, fluidity differences are evident as a function of the gel to liquid crystalline transition temperatures. Solubility of phospholipids is intimately linked to the confirmation of the aggregate material rather than strictly a chemical function of the molecule. Monoacyl phospholipids, which tend to form micelles, are usually more readily soluble in aqueous solutions. ${ }^{17}$

Sugar, polyols and their polymers: Although sugars and related compounds are highly water soluble and have few, if any, toxicity issues, they are less suitable than other carriers for the manufacture of solid dispersions. The melting point of most sugars is high, making preparation by the hot melt method problematic, and their solubility in most organic solvents is poor, making it difficult to prepare coevaporates. Even with these drawbacks, several attempts have been reported to prepare solid dispersions using sugars and their derivatives. Mannitol, which has a melting point of $165-168^{\circ} \mathrm{C}$ and decomposes only above $2500^{\circ} \mathrm{C}$, can be employed in some cases to prepare dispersions by the hot melt method. ${ }^{18}$

Organic acids and their derivatives: Organic acids such as succinic acid and citric acid have also been used as carriers in solid dispersions, originally to enhance the release rate of griseofulvin method.

Cyclodextrins: Cyclodextrins are primarily used to enhance solubility, chemical protection, taste masking and improved handling by the conversion of liquids into solids by entrapment.

Advantages of Cyclodextrins:

a. Increasing the stability of the drug

b. Release profile during gastrointestinal

c. Transit through modification of drug

d. Release site and time profile

e. Decreasing local tissue irritation.

f. Masking unpleasant taste.

\section{Methods for preparing solid solutions}

There are various manufacturing methods for solid dispersions that have been reported in literature. These are given below in Figure 1.

\section{Kneading technique}

In this method, carrier is permeated with water and transformed to paste. Drug is then added and kneaded for particular time. The kneaded mixture is then dried and passed through sieve if necessary. ${ }^{19}$

\section{Solvent method}

This method is also known as solvent evaporation method in which physical mixture of the drug and the carrier is dissolved in common solvent and is evaporated until a clear solvent free film is obtained. The main advantage is that the thermal decomposition of the drug or the carrier can be prevented because the organic solvents require a low temp for evaporation. The disadvantage in this method is difficulty in removing the solvent and higher cost of preparation. ${ }^{20}$ 


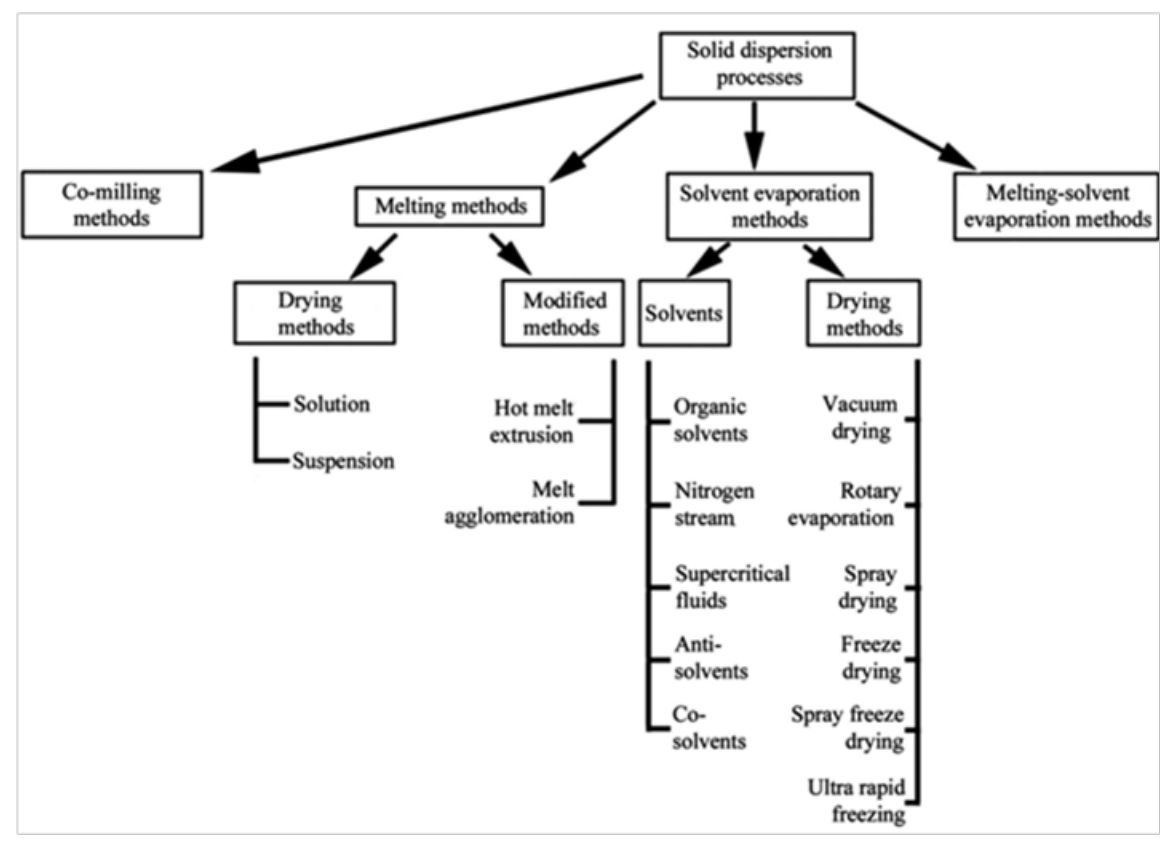

Figure I Different strategy for planning of strong scatterings.

\section{Co-precipitation method}

Required amount of drug is added to the solution of carrier. The system is kept under magnetic agitation and protected from the light. The formed precipitate is separated by vacuum filtration and dried at room temperature in order to avoid the loss of the structure water from the inclusion complex. ${ }^{21}$

\section{Melting method}

The melting method is suitable for heat stable materials with low melting points. The basic principle of the method consists of melting together the drug and carrier at a temperature slightly above their eutectic point, mixing the liquefied components. It is then cooled to acquire a congealed mass. It is crushed and sieved. ${ }^{22}$

Ex. albendazole and urea solid dispersion was prepared by this method.

\section{Co-grinding method}

Physical mixture of drug and carrier is mixed for some time employing a blender at a particular speed. The mixture is then charged into the chamber of a vibration ball mill steel balls are added. The powder mixture is pulverized. Then the sample is collected and kept at room temperature in a screw capped glass vial until use. Ex. chlordiazepoxide and mannitol solid dispersion was prepared by this method. ${ }^{23}$

\section{Gel entrapment technique}

Hydroxyl propyl methyl cellulose is dissolved in organic solvent to form a clear and transparent gel. Then drug for example is dissolved in gel by sonication for few minutes. Organic solvent is evaporated under vacuum. Solid dispersions are reduced in size by mortar and sieved. ${ }^{24}$

\section{Spray-drying method}

Drug is dissolved in suitable solvent and the required amount of carrier is dissolved in water. Solutions are then mixed by sonication or other suitable method to produce a clear solution, which is then spray dried using spray dryer. ${ }^{22}$

\section{Lyophilization technique}

Freeze-drying involves transfer of heat and mass to and from the product under preparation. This technique was proposed as an alternative method to solvent evaporation. Lyophilization has been thought of a molecular mixing technique where the drug and carrier are co dissolved in a common solvent, frozen and sublimed to obtain a lyophilized molecular dispersion..$^{24}$

\section{Electrospinning method}

In this technique electric force is used to withdraw a nano size fibre thread from the polymer sol/polymer melt. This a combination of solid dispersion with nanotechnology use in polymer industry. Stream of Polymer solution /melt is subjected to electric force (5 to $30 \mathrm{kv}$ ) which cause body of the liquid becomes charged, and electrostatic repulsion counteracts the surface tension. This made a strong cohesive force between the particle or droplets of polymer and a stream of fibre is formed. Then thinning and stretching of fibre to nano diameter is done by using whipping process called electrostatic repulsion lead to formation of uniform fibre in nano diameter. This process all depend on rate of feeding surface tension and electric force used. ${ }^{25}$

\section{Dropping method solution}

The dropping method, developed to facilitate the crystallization of different chemicals, is a new procedure for producing round particles from melted solid dispersions. This technique may overcome some of the difficulties inherent in the other methods. For laboratory-scale 
preparation, a solid dispersion of a melted drug-carrier mixture is pipette and then dropped onto a plate, where it solidifies into round particles. The use of carriers that solidify at room temperature may aid the dropping process. The dropping method not only simplifies the manufacturing process, but also gives a higher dissolution rate. It does not use organic solvents and, therefore, has none of the problems associated with solvent evaporation. ${ }^{26}$

\section{Melt extrusion method}

Solid dispersion by this method is composed of active ingredient and carrier, and prepare by hot-stage extrusion using a co-rotating twin-screw extruder. The concentration of drug in the dispersions is always $40 \%(\mathrm{w} / \mathrm{w})$. Melt extrusion technique is used in the preparation of diverse dosage forms in the pharmaceutical industry e.g. sustainedrelease pellets. ${ }^{27}$

\section{Melt agglomeration process}

This technique has been used to prepare Solid Dispersion where the binder acts as a carrier. $\mathrm{SD}(\mathrm{s})$ are prepared either by heating the binder, drug and excipient to a temperature above the melting point of the binder or by spraying a dispersion of drug in molten binder on the heated excipient by using a high shear mixer. A rotary processor has been shown to be alternative equipment for melt agglomeration because of easier control of the temperature and because higher binder content can be incorporated in the agglomerates.

\section{Super critical fluid (SCF) technology}

SCF is a substance above its critical temperature and pressure Critical point represents the highest temperature and pressure at which the substance exists as vapour and liquid in equilibrium. In this technique SCF is used to form solid dispersion of insoluble material/ polymer with drug cause increase in dissolution property. It is superior over conventional technique(spray drying, hot melt etc.), in this technique SCF carbon dioxide is mainly used which cause very rapid precipitation of solid mixture giving no time for separation of drug and polymer in preparation of solid dispersion. It form very stable small particle with higher surface area for good flow and low organic solvent residual. In recent Solid dispersion of carbamazepine with PEG-4000 are made using SCF carbon dioxide in precipitation vessel. Resulting in formation of carbamazepine with increase rate and extent of dissolution with low solvent residual.

\section{Characterization of solid dispersion}

A combination of two or more techniques is required to study its complete picture.

\section{Drug -carrier miscibility}

a. Hot stage microscopy

b. Differential scanning calorimetry

c. Powder X-ray diffraction

d. NMR 1H Spin lattice relaxation time

\section{Drug carrier interactions}

a. FT-IR spectroscopy

b. Raman spectroscopy

c. Solid state NMR

\section{Physical Structure}

a. Scanning electron microscopy

b. Surface area analysis

c. Surface properties

d. Dynamic vapor sorption

e. Inverse gas chromatography

f. Atomic force microscopy

g. Raman microscopy

\section{Amorphous content}

a. Polarised light optical microscopy

b. Hot stage microscopy

c. Humidity stage microscopy

d. DSC (MTDSC)

e. ITC

f. Powder X-ray diffraction

\section{Stability}

a. Humidity studies

b. Isothermal Calorimetry

c. $\mathrm{DSC}(\mathrm{Tg}$, Temperature recrystallization)

d. Dynamic vapor sorption

e. Saturated solubility studies

\section{Dissolution enhancement}

a. Dissolution

b. Intrinsic dissolution

c. Dynamic solubility

d. Dissolution in bio-relevant media

Powder X-ray diffraction can be used to qualitatively to detect the presence of crystalline forms in solid dispersion and to determine factors influencing recrystallization during storage stability studies. Sharper diffraction peaks indicate more crystalline material. ${ }^{28}$ In Infrared spectroscopy (IR) you can detect the variation in the energy distribution of interactions between drug and matrix. Sharp vibrational bands indicate crystallinity. Fourier Transformed Infrared Spectroscopy (FTIR) was used to accurately detect crystallinity ranging from 1 to $99 \%$ in pure material. ${ }^{29}$

Water vapour sorption can be used to discriminate between amorphous and crystalline material when the hygroscopicity is different. This method requires accurate data on the hygroscopicity of both completely crystalline and completely amorphous samples. Dissolution calorimetry measures the energy of dissolution, which is dependent on the crystallinity of the sample. Usually, dissolution of crystalline material is endothermic, whereas dissolution of amorphous material is exothermic. ${ }^{30}$

Macroscopic techniques that measure mechanical properties that 
are different amorphous and crystalline material can be indicative for the degree of crystallinity. Density measurements and Dynamic Mechanical Analysis (DMA) determine the modulus of elasticity for and viscosity and thus affected by the degree of crystallinity. However, also these techniques require knowledge about the additivity of these properties in intimately mixed binary solids. ${ }^{29}$ In Differential Scanning Calorimetry (DSC) samples are heated with a constant heating rate and the amount of energy necessary for that is detected. With DSC the temperatures at which thermal events occur can be detected. Thermal events can be a glass to rubber transition, (re)crystallization, melting or degradation. Furthermore, the melting- and (re)crystallization energy can be quantified. The melting energy can be used to detect the amount of crystalline material.

\section{In vitro dissolution studies}

In vitro dissolution studies are done for the find out dissolution behavior. The in-vitro dissolution study can be used to demonstrate the bioavailability or bioequivalence of the drug product through in vitro-in vivo correlation (IVIVC). On the other hand if absorption of the drug is dissolution rate limited that means the drug in the gastrointestinal fluid passes freely through the bio-membranes at a rate higher than it dissolves or is released from the dosage form. The specifically designed in-vivo dissolution study will be required in solid dispersion system to access the absorption rate, and hence its bioavailability and to demonstrate the bioequivalence ultimately. There are some apparatus used in United States pharmacopoeia for dissolution testing these are following. ${ }^{31}$

\section{Conclusion}

As with increasing number of poorly soluble drug candidates the need for improvements in the drug manufacturing technology also increases. Therefore solid dispersion technology seems to be a promising solution for improving the dissolution characteristics of such drugs. Aspects that still need to be addressed in the next years include further improvements in manufacturing on a large scale, and better predictions of whether a particular drug/carrier combination will lead to a true solid solution or to a partly crystalline dispersion as well as whether the dispersion will remain physically stable during further processing and storage. Last but not least, although this article has been devoted to the use of solid dispersions for the improvement of the release rate and oral bioavailability, by judicious choice of the carrier it is also possible to delay or slow down the release pattern of a drug by formulating it as a solid dispersion. The availability of a wide variety of polymers that are themselves poorly soluble or which swell under aqueous conditions suggests that solid dispersions have tremendous potential in the area of controlled release dosage forms.

\section{Acknowledgments}

$$
\text { None }
$$

\section{Conflicts of interest}

The author declares that there is no conflict of interest.

\section{References}

1. Aggarwal S, Gupta GD, Chaudhary S. Solid dispersion as an eminent strategic approach in solubility enhancement of poorly soluble drugs. Int $J$ Pharm Sci Res. 2010;1(8):1-13.
2. Delmar K, Bianco-Peled H. Composite chitosan hydrogels for extended release of hydrophobic drugs. Carbohydr Polym. 2016;136(1):570-580.

3. Velaga SP, Ghaderi R, Carlfors J. Preparation and characterization of hydrocortisone particles using a supercritical fluid extraction system. Int $J$ Pharm. 2002;231(2):155-166.

4. Lobenberg R, Amidon GL. Modern bioavailability, bioequivalence and biopharmaceutical classification system. New scientific approaches to international regulatory standards. Eur J Pharm Sci. 2000;50(1):3-20.

5. Blagden N, Gavan P, York P. Crystal engineering of active pharmaceutical ingredients to improve solubility and dissolution rates. Adv Drug Del Rev 2007;59(30):617-630.

6. Dhirendra K, Lewis S, Udupa N, et al. Solid Dispersions: A Review. Pak J Pharm Sci. 2009;22(2):234-246.

7. Giliyar C, Fikstad DT, Tyavanagimatt S. Challenges and opportunities in oral delivery of poorly water soluble drugs. Pharm Dev Technol. 2006;6:57-63.

8. Hsu CH, Cui Z, Mumper RJ, et al. Micellar Solubilization of Some Poorly Soluble Antidiabetic Drugs. AAPS Pharm Sci Tech. 2008;9(2):431-436.

9. Huang Y, Dai WG. Fundamental aspects of solid dispersion technology for poorly soluble drugs. Acta Pharm Sin B. 2014;4(1):18-25.

10. Hu J, Johnston KP, William RO. Spray freezing into liquid (SFL) Particle engineering technology to enhance dissolution of poorly water soluble drugs: organic solvent versus organic/aqueous co-solvent systems. Eur $J$ Pharm Sci. 2003;20(3):295-303.

11. Chaudhary A, Nagaich U, Gulati N, et al. Enhancement of solubilization and bioavailability of poorly soluble drugs by physical and chemical modifications. J Adv Pharm Technol Res. 2012;2(1):32-67.

12. Cong $\mathrm{W}$, Shen $\mathrm{L}, \mathrm{Xu} \mathrm{D}$ et al. Solid dispersion tablets of breviscapine with polyvinylpyrrolidone $\mathrm{K} 30$ for improved dissolution and bioavailability to commercial breviscapine tablets in beagle dogs. Eur J Drug Metab Pharmacokinet. 2014;39(3):203-210.

13. Shah H, Shah V, Bhutani S, et al. Dissolution improvement of nebivolol hydrochloride using solid dispersion adsorbate technique. Asian J Pharm. 2015;21(2):49-55.

14. Liebenberg W, Villiers MM, Wurster DE, et al. The effect of polymorphism on powder compaction and dissolution properties of chemically equivalent oxytetracycline hydrochloride powders. Drug Dev Ind Pharm. 1999;25(9):1027-1033.

15. Pandya RB, Mehta TA, Gohel MC. Solid dispersion adsorbate-a novel technique for dissolution enhancement of febuxostat. Int J Pharm Sci Res. 2015;6(10):4236-4242.

16. Pouton CW. Formulation of poorly water soluble drugs for oral administration :physicochemical and physiological issues and the lipid formulation classification system. Eur J Pharm Sci. 2006;29(3-4):278-287.

17. Patrick JM, Tonglei L, Lynne ST. Estimation of Drug-Polymer Miscibility and Solubility in Amorphous Solid Dispersions Using Experimentally Determined Interaction Parameters. Pharm Res. 2009;26(1):133-139.

18. Sultana S, Saifuddin AHM. Solid dispersion currently practiced in pharmaceutical field. IJOART. 2016;5(3):170-175.

19. Janssens S, Mooter GV. Review: physical chemistry of solid dispersions. $J$ Pharm Pract. 2009;61(12):1571-1586.

20. Hulsmann S, Backensfeld T, Keitel S, et al. Melt extrusion-an alternative method for enhancing the dissolution rate of $17 \beta$-estradiol hemihydrate. Eur J Pharm Biopharm. 2000;49:237-242. 
21. Rambali B, Verreck G, Baert L, et al. Itraconazole formulation studies of the melt- extrusion process with mixture design. Drug Dev Ind Pharm. 2003;29(6):641-652.

22. Serajuddin ATM. Solid dispersion of poorly water-soluble drugs: early promises, subsequent problems, and recent breakthroughs. J Pharm Sci. 1999;88(10):1058-1066.

23. Sharma C, Jain P. Preparation and characterization of solid dispersions of carvedilol with PVP K30. Res Pharm Sci. 2010;5(1):49-56.

24. Teja A, Musmade PB, Khade AB, et al. Simultaneous improvement of solubility and permeability by fabricating binary glassy materials of Talinolol with Naringin: Solid state characterization, in-vivo in-situ evaluation. Eur J Pharm Sci. 2015;78(1):234-244.

25. Kim JS, Kim MS, Park HJ, et al. Physiochemical properties and ora bioavailability of amorphous atrovastatin hemi-calcium using spray-drying and SAS process. Int J Pharm. 2008;359(1-2):211-219.
26. Kalepu S, Nekkanti V. Insoluble drug delivery strategies: review of recent advances and business prospects. Acta Pharm Sin B. 2015;5(5):442-453.

27. Leuner C, Dressman J. Improving drug solubility for oral delivery using solid dispersions. Eur J Pharm Biopharm. 2000;50(1):47-60.

28. Wang L, Cui FD, Sunada H. Preparation and evaluation of solid dispersions of nitrendipine prepared with fine silica particles using the melt-mixing method. Chem Pharm Bull. 2006;54(1):37-43.

29. Apparao B, Shivalingam MR, Reddy YVK, et al. Formulation and evaluation of aceclofenac solid dispersions for dissolution rate enhancement. IJPSDR. 2010;2(2):146-150.

30. Trespidi L, Soldato PD. Solid dispersions of nitrate active principles. A1: Patent application; 2004

31. Yu LX. A Biopharmaceutics classification System: The scientific Basis for Biowaiver Extensions. Pharm Res. 2002;19(7):921-925. 